\title{
Type of preadmission glucose-lowering treatment and prognosis among patients hospitalised with myocardial infarction: a nationwide follow-up study
}

\author{
H. T. Horsdal • S. P. Johnsen • F. Søndergaard • \\ J. Rungby
}

Received: 26 October 2007 / Accepted: 14 January 2008 / Published online: 19 February 2008

(C) Springer-Verlag 2008

\begin{abstract}
Aims/hypothesis We examined whether the type of preadmission glucose-lowering treatments explained differences in mortality rate and risk of readmission with myocardial infarction (MI) and heart failure following first-time hospitalisation for MI in patients with type 2 diabetes mellitus. Methods We conducted a nationwide population-based followup study among all Danish patients hospitalised with firsttime MI from 1996 to 2004. Data on use of glucose-lowering drugs and other medications, comorbidities, socioeconomic status, laboratory findings, readmission with MI and heart failure, and death were obtained from medical databases. We computed mortality rates and rates of MI and heart failure readmission, according to type of glucose-lowering treatment and used Cox's proportional hazards regression
\end{abstract}

H. T. Horsdal $(\bowtie) \cdot$ S. P. Johnsen $\cdot$ F. Søndergaard

Department of Clinical Epidemiology,

Aarhus University Hospital,

Ole Worms Allé 1150,

DK-8000 Aarhus, Denmark

e-mail: ht@dce.au.dk

H. T. Horsdal · J. Rungby

Department of Pharmacology, University of Aarhus,

Aarhus, Denmark

S. P. Johnsen

Center of Cardiovascular Research, Aalborg Hospital,

Aarhus University Hospital,

Aalborg, Denmark

J. Rungby

Department of Endocrinology C, Aarhus University Hospital,

Aarhus, Denmark analysis to compute hazard ratios (HRs) as estimates of relative risks.

Results We identified 8,494 MI patients with type 2 diabetes mellitus. The overall cumulative 30 day and 1 year mortality rates were 22.2 and $36.6 \%$, respectively. Patients not receiving any glucose-lowering drugs (adjusted 30 day HR: $0.79,95 \%$ CI: $0.57-1.10$ ) and users of any combination (adjusted 30 day HR: 1.43, 95\% CI: 0.98-2.09) had the lowest and highest mortality rates, respectively, when compared with users of sulfonylureas. We found that glycaemic control had no impact on the risk estimates in a subanalysis including biochemical laboratory data. We found no differences in the risk of new MI and heart failure between the different glucose-lowering agents.

Conclusions/interpretation Type of preadmission glucoselowering treatment in monotherapy is not associated with substantial differences in prognosis following hospitalisation with MI. However, patients treated with any combination had increased mortality rates.

Keywords Epidemiology $\cdot$ Glucose-lowering treatment . Heart failure · Hospitalisation · MI · Mortality · Myocardial infarction P Prognosis · Readmission . Type 2 diabetes mellitus

$\begin{array}{ll}\text { Abbreviations } \\ \text { CK-MB } & \text { creatine kinase-myocardial band } \\ \text { HR } & \text { hazard ratio } \\ \text { HRT } & \text { hormone replacement therapy } \\ \text { ICD } & \text { International Classification of Disease } \\ \text { IDA } & \begin{array}{l}\text { Integrated Database for Labour } \\ \text { Market Research }\end{array} \\ \text { MI } & \begin{array}{l}\text { myocardial infarction } \\ \text { PCOS }\end{array} \\ \end{array}$




\section{Introduction}

Type 2 diabetes mellitus is a progressive disease characterised by varying degrees of insulin resistance and relative insulin deficiency. Initially, glycaemic control is achieved non-pharmacologically (i.e. by diet and exercise), but in later stages pharmacotherapy with one or more drug is required [1]. It is likely that treatments aimed to improve glycaemic control will prevent cardiovascular complications, which are often seen among patients with type 2 diabetes mellitus, but it remains uncertain whether the treatments themselves have adverse effects on the cardiovascular system.

Patients with diabetes mellitus have a poorer prognosis after myocardial infarction (MI) including increased rates of new MI, heart failure and death, when compared with their non-diabetic counterparts [2]. Data regarding outcome in cardiac patients using various types of glucose-lowering drugs are sparse and conflicting [3-14], and the existing studies have some limitations, including small and often nonrepresentative samples and shortcomings in the data analysis.

As a large proportion of patients with type 2 diabetes mellitus suffer from cardiovascular diseases and are permanently dependent on their glucose-lowering drugs, it is important to clarify the risk associated with the different glucose-lowering drugs. Here, in a nationwide populationbased follow-up study, we examine the prognosis among Danish patients with type 2 diabetes mellitus, who were admitted to hospital with a first-time MI and were using various types of glucose-lowering treatments.

\section{Methods}

Setting and design We conducted this follow-up study within the entire Danish population (approximately 5.3 million).

The Danish National Health Service provides tax-supported healthcare for all inhabitants, guaranteeing free access to general practitioners and hospitals, and refunding a variable proportion of the prescription medication costs. The Danish Civil Registration System keeps electronic records on sex, date of birth, change of address, date of emigration and changes in vital status since $1968[15,16]$. The records carry a unique ten-digit civil registration number, assigned to every Danish citizen and used in all Danish registries, enabling unambiguous linkage between them.

Patients with MI The Danish National Patient Registry [17], established in 1977, collects data on all hospitalisations from non-psychiatric hospitals in the country, including dates of admission and discharge, surgical procedure(s) performed and up to 20 discharge diagnoses assigned by the treating physician and coded according to the International Classification of Diseases (ICD) 8th revision (ICD-8) until the end of 1993 (thereafter 10th revision [ICD-10]). From the registry, we identified all patients with a first-time primary discharge diagnosis of MI (ICD-10 codes I21.0-I21.9) among those who had been hospitalised between 1 January 1996 and 30 November 2004.

Glucose-lowering agents The Register of Medicinal Product Statistics contains data from 1995 onwards on all prescription drugs dispensed at all Danish pharmacies, including patients' civil registration numbers, type of drug according to the Anatomical Therapeutic Chemical classification system and date of drug dispensing.

In Denmark, glucose-lowering drugs are available by prescription only. Among the MI patients, we traced all prescriptions for glucose-lowering drugs redeemed prior to admission for MI. Users of glitazones $(n=2)$, meglitinides $(n=60)$ and $\alpha$-glucosidase inhibitors $(n=19)$ were excluded from the analyses due to the low number of treated patients.

Patients who had used only one type of glucose-lowering drug in the 90 days prior to hospitalisation were categorised according to the class of the glucose-lowering drug: sulfonylureas, metformin or insulin. Patients who had used more than one type of glucose-lowering drug during the 90 days prior to hospitalisation were categorised as combined users and divided according to the type of combination: sulfonylureas and metformin, sulfonylureas and insulin, metformin and insulin, or sulfonylureas, metformin and insulin. Patients with a diagnosis of diabetes since 1977 according to the Patient Registry (ICD-8 codes 249, 250, ICD-10 codes E10, E11, E14, G63.2, H36.0, N08.3) but not using any glucose-lowering drugs during the 90 days prior to hospitalisation were categorised as diabetic patients without pharmacotherapy. For all patients, we also received information on whether they had used other types of glucose-lowering drugs before the 90 days prior to the admission for MI, as well as on their use of glucose-lowering drugs within 1 year after the admission.

We classified the diabetic patients according to their type of diabetes: type 1 if they were less than 30 years old by the time of the first prescription or diagnosis and had never received a prescription for an oral glucose-lowering drug; type 2 if they had not received pharmacotherapy or had received a prescription for an oral glucose-lowering drug or were older than 30 years at the time of first prescription or diagnosis, regardless of treatment. Patients with type 1 diabetes mellitus $(n=175)$ were excluded from the study.

Patients with polycystic ovary syndrome (PCOS) may also receive treatment with metformin, thus to avoid inclusion of these non-diabetic patients, we sought to identify patients with a PCOS diagnosis (ICD-10 code E28.2) among the users of metformin. No such patients were identified.

Endpoints The endpoints were 30 day and 1 year all-cause mortality (in and outside the hospital) and readmission with 
new MI or heart failure within 1 year after admission with MI. Mortality was ascertained from the Civil Registration System; readmission with a new MI (ICD-10 codes I21, I22) or heart failure (ICD-10-codes I11.0, I13.0, I13.2, I25.5, I42.0, I42.6, I42.7, I42.8, I42.9, I50.0, I50.1, I50.9) were ascertained from the Danish National Patient Registry. Readmission with MI within 28 days of the original MI was not considered a new event [18].

Covariates We obtained data on covariates from the Danish National Patient Registry, the Register of Medicinal Product Statistics, the Integrated Database for Labour Market Research (IDA) at Statistics Denmark [19] and, for a subset, from the Laboratory Information Systems in the Counties of North Jutland and Aarhus.

Based on discharge diagnoses from the Danish National Patient Registry, we computed, for each patient, the comorbidity index score developed by Charlson et al. [20]. The index covers 19 major disease categories, including diabetes mellitus, MI, heart failure, cerebrovascular diseases and cancer, weighted according to their impact on patient survival. MI and diabetes mellitus were excluded from the comorbidity index calculations. We also obtained information on previous diagnoses of hypertension, coronary revascularisation procedures (percutaneous coronary intervention and coronary artery bypass graft), alcoholismrelated diseases and diabetes complications (i.e. retinopathy, nephropathy and neuropathy). Data on coronary revascularisation procedures performed during or after the admission for MI were also obtained.

We identified all prescriptions for cardiovascular drugs (antihypertensive drugs, lipid-lowering drugs, platelet inhibitors, vitamin $\mathrm{K}$ antagonists) and hormone replacement therapy (HRT) redeemed before the date of admission for MI or within 1 year after the admission.

We estimated the duration of diabetes as the time since the earliest prescription for a glucose-lowering drug or the earliest diabetes diagnosis and categorised the duration in three groups: $\leq 5$ years, 5 to 10 years, $>10$ years. Finally, based on data from IDA, we classified the patients according to socioeconomic status (employed, pensioner or other) in the year prior to admission for MI.

Biochemical data reflecting the intensity of the antiglycaemic treatment and the extent of myocardial damage following admission with MI were available from the Laboratory Information System for tests analysed in North Jutland and Aarhus Counties, covering a population of approximately $1,150,000$ ( $22 \%$ of the total Danish population). Data were available on laboratory tests from all the counties' hospitals and general practices. We retrieved data on $\mathrm{HbA}_{1 \mathrm{c}}$, blood glucose, troponin $\mathrm{T}$ and creatine kinase-myocardial band (CK-MB). We used the latest measurement of $\mathrm{HbA}_{1 \mathrm{c}}$ within 180 days prior to admission and 7 days after the admission and the highest level of blood glucose, troponin $\mathrm{T}$ and CK-MB on the day of admission or the following day.

As the proportion of users of the different glucoselowering drugs varied during the study period, thereby creating a risk of comparing patients from different calendar periods when comparing the treatments, we also included the calendar time of admission in the analyses.

Statistical analyses Characteristics of the users of various types of glucose-lowering treatments were compared using a $\chi^{2}$ analysis for the categorical variables, whereas continuous variables were compared using Kruskal-Wallis tests.

Follow-up began on the date of admission with MI and ended on the date of admission with new MI or heart failure (only in analyses on risk of new MI or heart failure, respectively), death, emigration or after 30 days or 1 year. We computed Kaplan-Meier mortality curves for the glucoselowering treatments and computed the cumulative 30 day and 1 year mortality rates.

We used Cox's proportional hazards regression analysis (with the Efron approximation to handle tied survival times) to compute hazard ratios (HRs) as estimates of the relative risks for each outcome. We included all the measured covariates; use of glucose-lowering drugs, cardiovascular drugs, HRT and coronary revascularisation procedures after MI were treated as time-dependent covariates. The $p$ values were estimated by Wald tests. The largest group, i.e. patients receiving sulfonylureas, served as the reference group in all analyses.

To examine possible sex-related differences in prognosis, we also analysed the HRs stratified by sex. Differences in prognosis between the different combination types were assessed by likelihood ratio tests; differences in troponin $\mathrm{T}$ and CK-MB levels, which may reflect infarct size [21], were assessed by Kruskal-Wallis tests.

Prescriptions for glucose-lowering drugs in Denmark are usually issued for 3 months, but may be issued for up to 6 months. We therefore also analysed the data based on drug use within 180 days prior to hospitalisation.

We analysed data with Stata 8.2 (StataCorp LP, College Station, TX, USA) and with version 9.13 of SAS software (SAS Institute, Cary, NC, USA). The statistical significance level was set to 0.05 in all analyses.

\section{Results}

Diabetes treatment groups We identified 8,494 patients with type 2 diabetes mellitus who were hospitalised with a firsttime MI during the study period. Among these patients, $31.7 \%$ were being treated with sulfonylureas, $6.0 \%$ with metformin, $21.5 \%$ with insulin and $15.7 \%$ with any combination of glucose-lowering drugs, while $25.1 \%$ were not 
Table 1 Descriptive characteristics according to glucose-lowering treatment of patients with type 2 diabetes mellitus hospitalised with a first-time MI in Denmark during the period 1996 to 2004

\begin{tabular}{|c|c|c|c|c|c|c|c|c|c|c|c|}
\hline \multirow[t]{3}{*}{ Characteristic $^{a}$} & \multirow{2}{*}{\multicolumn{2}{|c|}{$\frac{\mathrm{SU}}{(n=2,691)}$}} & \multirow{2}{*}{\multicolumn{2}{|c|}{$\frac{\text { Metformin }}{(n=511)}$}} & \multirow{2}{*}{\multicolumn{2}{|c|}{$\begin{array}{l}\text { Insulin } \\
(n=1,827)\end{array}$}} & \multirow{2}{*}{\multicolumn{2}{|c|}{$\begin{array}{l}\text { Combination } \\
(n=1,333)\end{array}$}} & \multirow{2}{*}{\multicolumn{2}{|c|}{$\begin{array}{l}\text { No pharmacotherapy } \\
(n=2,132)\end{array}$}} & \multirow[t]{3}{*}{$p$ value } \\
\hline & & & & & & & & & & & \\
\hline & $n$ & $\%$ & $n$ & $\%$ & $n$ & $\%$ & $n$ & $\%$ & $n$ & $\%$ & \\
\hline Age in years, median (range) & 75.4 & $36-100$ & 67.5 & $33-96$ & 71.9 & $25-96$ & 70.1 & $37-97$ & 74.1 & $35-100$ & $<0.001$ \\
\hline Sex (men) & 1,583 & 58.8 & 324 & 63.4 & 932 & 51.0 & 763 & 57.2 & 1,218 & 57.1 & $<0.001$ \\
\hline Calendar period & & & & & & & & & & & $<0.001$ \\
\hline $1996-1998$ & 911 & 33.8 & 73 & 14.3 & 527 & 28.9 & 336 & 25.2 & 555 & 26.0 & \\
\hline 1999-2001 & 909 & 33.8 & 153 & 29.9 & 585 & 32.0 & 398 & 29.9 & 738 & 34.6 & \\
\hline $2002-2004$ & 871 & 32.4 & 285 & 55.8 & 715 & 39.1 & 599 & 44.9 & 839 & 39.4 & \\
\hline Duration of diabetes mellitus (years) & & & & & & & & & & & $<0.001$ \\
\hline$\leq 5$ & 1,702 & 63.2 & 346 & 67.7 & 322 & 17.6 & 522 & 39.2 & 1,198 & 56.2 & \\
\hline $5-10$ & 707 & 26.3 & 122 & 23.9 & 571 & 31.3 & 544 & 40.8 & 557 & 26.1 & \\
\hline$>10$ & 282 & 10.5 & 43 & 8.4 & 934 & 51.1 & 267 & 20.0 & 377 & 17.7 & \\
\hline Charlson's comorbidity index & & & & & & & & & & & $<0.001$ \\
\hline 0 & 1,296 & 48.1 & 294 & 57.5 & 624 & 34.2 & 682 & 51.2 & 822 & 38.6 & \\
\hline $1-2$ & 1,065 & 39.6 & 186 & 36.4 & 877 & 48.0 & 522 & 39.1 & 930 & 43.6 & \\
\hline$\geq 3$ & 330 & 12.3 & 31 & 6.1 & 326 & 17.8 & 129 & 9.7 & 380 & 17.8 & \\
\hline Discharge diagnoses of & & & & & & & & & & & \\
\hline Hypertension & 1,999 & 74.3 & 393 & 76.9 & 1,471 & 80.5 & 1,060 & 79.5 & 1,635 & 76.7 & $<0.001$ \\
\hline Alcoholism & 71 & 2.6 & 19 & 3.7 & 102 & 5.6 & 41 & 3.1 & 111 & 5.2 & $<0.001$ \\
\hline Former revascularisation & 80 & 3.0 & 25 & 4.9 & 93 & 5.1 & 45 & 3.4 & 91 & 4.3 & 0.003 \\
\hline Retinopathy & 135 & 5.0 & 21 & 4.1 & 594 & 32.5 & 156 & 11.7 & 194 & 9.1 & $<0.001$ \\
\hline Nephropathy ${ }^{\mathrm{b}}$ & 95 & 3.5 & 19 & 3.7 & 301 & 16.5 & 81 & 6.1 & 169 & 7.9 & $<0.001$ \\
\hline Neuropathy & 110 & 4.1 & 26 & 5.1 & 342 & 18.7 & 107 & 8.0 & 139 & 6.5 & $<0.001$ \\
\hline Prescription for ${ }^{\mathrm{c}}$ & & & & & & & & & & & \\
\hline Antihypertensive drugs & 1,972 & 73.3 & 385 & 75.3 & 1,446 & 79.2 & 1,047 & 78.5 & 1,594 & 74.8 & $<0.001$ \\
\hline Platelet inhibitors & 562 & 20.9 & 111 & 21.7 & 424 & 23.2 & 311 & 23.3 & 401 & 18.8 & $<0.001$ \\
\hline Vitamin $\mathrm{K}$ antagonists & 88 & 3.3 & 16 & 3.1 & 68 & 3.7 & 60 & 4.5 & 66 & 3.1 & 0.209 \\
\hline Lipid-lowering drugs & 316 & 11.7 & 140 & 27.4 & 352 & 19.3 & 299 & 22.4 & 273 & 12.8 & $<0.001$ \\
\hline HRT & 243 & 9.0 & 46 & 9.0 & 194 & 10.6 & 138 & 10.4 & 220 & 10.3 & 0.339 \\
\hline Socioeconomic status ${ }^{\mathrm{d}}$ & & & & & & & & & & & $<0.001$ \\
\hline Employed & 285 & 10.8 & 114 & 22.7 & 204 & 11.4 & 184 & 14.0 & 258 & 12.3 & \\
\hline Pensioner & 2,287 & 86.8 & 364 & 72.5 & 1,535 & 86.1 & 1,068 & 81.3 & 1,774 & 84.6 & \\
\hline Other & 63 & 2.4 & 24 & 4.8 & 45 & 2.5 & 62 & 4.7 & 66 & 3.1 & \\
\hline Laboratory data $\mathrm{e}^{\mathrm{e}}$ & & & & & & & & & & & \\
\hline $\mathrm{HbA}_{1 \mathrm{c}}, \%(\text { median [range] })^{\mathrm{f}}$ & 7.5 & $4.7-12.5$ & 7.2 & $5.4-11.3$ & 8.5 & $5.4-13.9$ & 8.1 & $1.0-13.8$ & 7.1 & $4.7-13.1$ & $<0.001$ \\
\hline 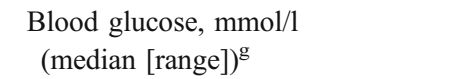 & 12.8 & $3.9-48.4$ & 10.9 & $5.6-26.3$ & 14.8 & $2.3-61.7$ & 14.4 & $4.8-36.1$ & 10.8 & $3.2-33.9$ & $<0.001$ \\
\hline Troponin $\mathrm{T}, \mu \mathrm{g} / 1\left(\right.$ median $[\text { range] })^{\mathrm{h}}$ & 1.29 & $0.01-64$ & 0.96 & $0.01-50$ & 1.42 & $0.01-48$ & 1.2 & $0.01-34$ & 1.00 & $0.01-43$ & $<0.001$ \\
\hline $\begin{array}{l}\text { Creatine kinase } \mathrm{MB}, \mu \mathrm{g} / \mathrm{l} \\
\left(\text { median }[\text { range] })^{\mathrm{i}}\right.\end{array}$ & 42.4 & $0.8-1746$ & 35.6 & $0.3-455$ & 41.6 & $1.5-1000$ & 31.1 & $1.9-529$ & 29.9 & $0.1-1000$ & $<0.001$ \\
\hline
\end{tabular}

${ }^{a}$ All variables are shown as $n(\%)$, except for age and laboratory data, which are shown as median (range)

${ }^{\mathrm{b}}$ Also included in the Charlson index

${ }^{c}$ Received a prescription within 90 days (platelet inhibitors and vitamin $\mathrm{K}$ antagonists) or ever (all others) before admission for MI

dTe year prior to admission for MI

e Data only available for patients from North Jutland and Aarhus Counties

${ }^{\mathrm{f}}$ Measured within 180 days prior to admission and 7 days after admission with MI ( $n=1,189, n=265, n=352, n=69, n=323, n=180$ for total, no pharmacotherapy, sulfonylurea, metformin, insulin, combination treatments, respectively)

${ }^{\mathrm{g}}$ Highest measurement at the day of admission $(n=1,403, n=345, n=424, n=76, n=348, n=210$ for total, no pharmacotherapy, sulfonylurea, metformin, insulin, combination treatments, respectively)

${ }^{h}$ Highest measurement at the day of admission or the following day $(n=1,043, n=270, n=307, n=67, n=246, n=153$ for total, no pharmacotherapy, sulfonylurea, metformin, insulin, combination treatments, respectively)

${ }^{\mathrm{i}}$ Highest measurement at the day of admission or the following day $(n=770, n=206, n=214, n=50, n=188, n=112$ for total, no pharmacotherapy, sulfonylurea, metformin, insulin, combination treatments, respectively)

$\mathrm{SU}$, sulfonylurea 
receiving glucose-lowering pharmacotherapy at the time of admission. The main clinical and laboratory characteristics of the patients according to glucose-lowering treatment are presented in Table 1.

All-cause mortality rate Cumulative mortality curves for the different treatment groups are shown in Fig. 1. We excluded 161 patients for whom data on socioeconomic status were missing. A total of 1,851 of the remaining $8,333(22.2 \%$, 95\% CI: 21.3-23.1) patients died within 30 days after admission. Within 1 year, $36.6 \%$ (95\% CI: $35.5-37.7)$ of the patients were dead.

Compared with users of sulfonylureas, we found lower crude risks of mortality among patients using metformin (30 day HR: $0.55,95 \%$ CI: $0.43-0.71$ ) or any combination (30 day HR: $0.85,95 \%$ CI: $0.73-0.98$ ). In contrast, the crude 30 day HRs were 1.10 (95\% CI: $0.97-1.24)$ and 0.99 (95\% CI: 0.88-1.11) for users of insulin and patients not receiving pharmacotherapy, respectively, compared with users of sulfonylureas. After adjustment for differences in covariates, we found no differences between the glucose-lowering drugs in monotherapy i.e. the 30 day adjusted HRs for the use of metformin and insulin were 0.85 (95\% CI: 0.40-1.81) and 1.05 (95\% CI: $0.63-1.76$ ), but the use of any combination was associated with an increased risk of mortality compared with use of sulfonylureas (adjusted 30 day HR: $1.43,95 \% \mathrm{CI}$ : 0.98-2.09) (Table 2). The highest risk estimate was found among users of the triple combination with sulfonylurea, metformin and insulin (adjusted 30 day HR: 1.79, 95\% CI: 0.65-4.95), but we found no differences in mortality rate when comparing use of the different types of combination $(p=0.33)$. When estimating the 1 year HRs, we found similar results (Table 2).

For most treatments, the association was similar among men and women. However, among women the use of

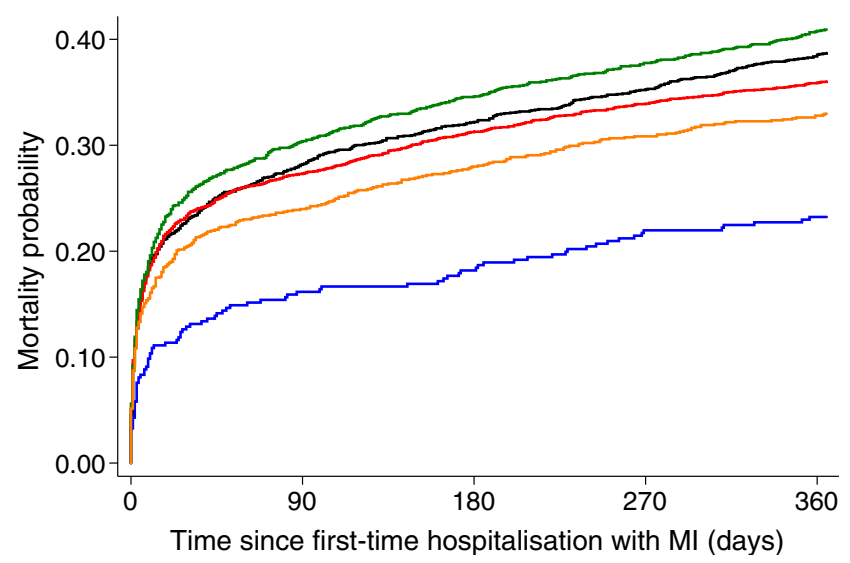

Fig. 1 Kaplan-Meier curves of 1 year all-cause mortality rate after hospitalisation with myocardial infarction (MI) according to use of glucose-lowering treatments in the 90 days prior to hospitalisation. Green line, insulin; black line, no pharmacotherapy; red line, sulfonylureas; orange line, any combination; blue line, metformin metformin was associated with a lower mortality rate than the use of sulfonylureas (adjusted 30 day HR: 0.51, 95\% CI: $0.25-1.04$; adjusted 1 year HR: $0.49,95 \% \mathrm{CI}: 0.30$ 0.79 ), whereas among men the risk appeared to be increased (adjusted 30 day HR: 1.80, 95\% CI: 0.91-3.56; adjusted 1 year HR: $1.82,95 \%$ CI: $1.25-2.64$ ).

The results were similar when estimating the HRs based on drug use 180 days prior to MI.

New MI and heart failure Within 1 year of follow-up, 8.4 and $9.6 \%$ of the patients were readmitted with a new MI and heart failure, respectively. We found no substantial differences in the risks of new MI or heart failure between users of the different glucose-lowering treatments (Table 3), nor were there any differences in risk of new MI $(p=0.75)$ or heart failure $(p=0.28)$ among users of the different types of combination.

The results were similar when estimating the HRs based on drug use 180 days prior to MI.

Subanalysis including biochemical parameters In the North Jutland and Aarhus County subcohort, data on $\mathrm{HbA}_{1 \mathrm{c}}$ and admission blood glucose levels were available for 1,027 patients with MI. We examined the effect of the intensity of glycaemic control among this subset of patients, although with a much weaker statistical precision. The $\mathrm{HbA}_{1 \mathrm{c}}$ and admission blood glucose levels had only minor effects on the risk estimates, i.e. further adjustment for these parameters changed the estimates by only 1 to $6 \%$. Thus compared with use of sulfonylureas, the adjusted HR of 30 day mortality rate was 1.90 (95\% CI: $0.68-5.32)$ for use of any combination. After further adjustment for $\mathrm{HbA}_{1 \mathrm{c}}$ and blood glucose, the adjusted HR of 30 day mortality rate was 1.83 (95\% CI: 0.65-5.13). A similar pattern was found when estimating the 1 year HRs.

We found significant differences in the levels of CK-MB ( $p=0.0001)$ and troponin $\mathrm{T}(p=0.0001)$ between the users of different glucose-lowering treatments (Table 1). The highest levels were found among users of sulfonylureas and insulin, probably reflecting a larger infarct size in these patients.

\section{Discussion}

In this large population-based follow-up study, we found no substantial differences in the prognosis following hospitalisation with $\mathrm{MI}$ among patients with type 2 diabetes mellitus using different glucose-lowering drugs in monotherapy. However, use of any combination of glucose-lowering drugs was associated with an increased mortality rate.

The main strength of this study is the use of nationwide population-based registries with complete follow-up both during and after hospitalisation. As these data sources 


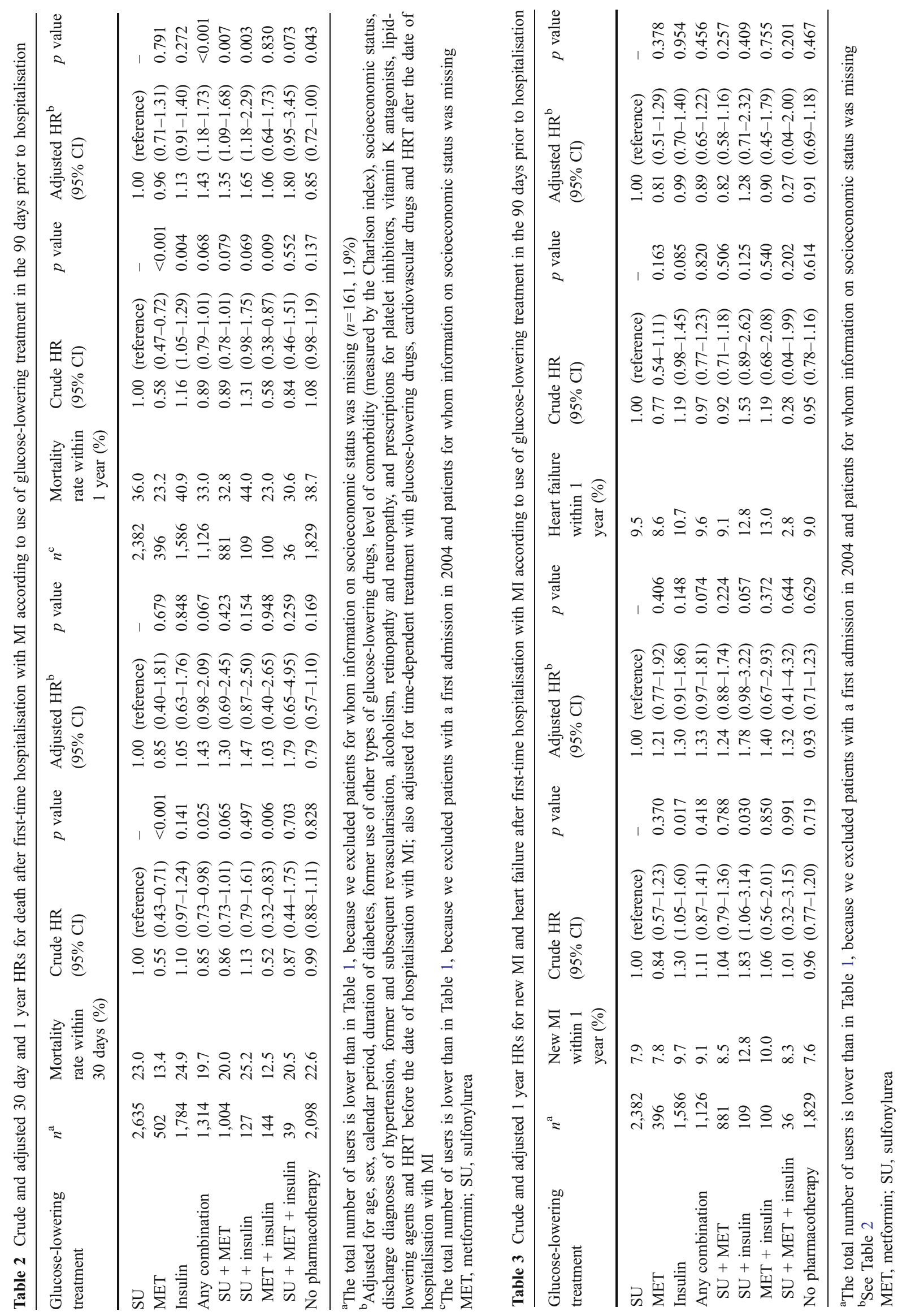


comprise complete population data, our results reflect the daily clinical practice in Denmark. Further, the use of nationwide registries with prospectively collected data minimises the risk of both selection and information bias. Another advantage is the ability to adjust for the wide range of possible confounding factors collected independently of hospitalisation with MI through access to the different medical registries.

The validity of our estimates depends on the accuracy of the registries. However, the validity of the hospital discharge diagnoses used to identify our study population of MI patients seems high [22], and the combined use of the Danish National Patient Registry and the Register of Medicinal Product Statistics to identify patients with diabetes mellitus has proven to be of high quality and nearly complete [23].

As we used the redemption of a prescription as a proxy for compliance, we may have overestimated actual exposure. Further, the lack of information on non-hospitalised MI cases, including patients who die before reaching the hospital, might introduce selection bias, if users of one particular glucose-lowering treatment were more likely to die from MI before hospitalisation.

Finally, despite our adjustment for a wide range of confounding factors, including intensity of the antiglycaemic treatment, we cannot entirely rule out the possibility of residual or unmeasured confounding related to differences in in-hospital treatment for MI, diet, smoking and obesity. In contrast, it could be argued that not all covariates should be considered true confounding factors, but rather intermediate steps in the association between use of a specific drug and prognosis (e.g. $\mathrm{HbA}_{1 \mathrm{c}}$ ). We may in theory have underestimated the real effect by adjusting for the covariates. However, in reality neither measures of intensity of glycaemic control, nor duration of diabetes nor other possible 'intermediate step covariates' had any substantial impact on the adjusted risk estimates.

Our results are consistent with a number of other studies on both short- and long-term mortality rate after admission with MI. Two studies found that the type of diabetes treatment in monotherapy at discharge had no significant association with 28 day [4] and 1 year mortality rate [5]. One of these also found similar risk of readmission with MI or heart failure within 1 year among MI patients discharged on metformin and non-insulin sensitisers (sulfonylureas or insulin) [5]. Another study showed no differences in longterm $(>3$ years) survival rate between patients treated with sulfonylureas prior to MI and those receiving any other glucose-lowering treatments (diet, insulin or metformin and/or acarbose) [6]. Further, in agreement with our finding, an increased mortality rate has also previously been found among users of combination therapy (sulfonylureas and metformin) when compared with patients treated with diet, whereas no increased mortality rate was found among users of the drugs in monotherapy [14].

Recently, we found some indications of variation in 30 day mortality rates after MI among users of different glucose-lowering treatments [7]. However, the study was small (patients with diabetes $n=867$ ) with few data on confounding factors, making it difficult to interpret the size of the variation.

Other studies have also implied that use of specific glucose-lowering drugs is associated with an adverse prognosis among patients with previous coronary artery disease. Both sulfonylureas $[3,8,9]$, metformin $[9,10]$ and insulin $[9,11-13]$ have been associated with increased mortality rate compared with diet. However, this might reflect a longer duration of diabetes and thus a more advanced stage of diabetes and/or a poorer glycaemic control among users of these drugs, rather than actual drug effects.

The finding that metformin carried a significantly lower risk in women than in men remains unexplained and may be due to chance. However, it has recently been reported that high proinsulin levels (which are reduced by metformin [24]) are pro-atherosclerotic in women, but not in men [25], and this might provide at least some explanation for our finding.

Although this study has shown similar effects of different glucose-lowering drugs in monotherapy prior to MI on the prognosis after the MI, concerns about differences in the risk of developing cardiovascular disease, including MI, among users of different glucose-lowering drugs remain [7, 26-29].

In conclusion, the prognosis after MI does not seem to vary substantially according to the glucose-lowering drugs used in monotherapy prior to MI. However, use of any combination seems to be related to an increased mortality rate.

Acknowledgements This study was supported by the Research Initiative of Aarhus University Hospital, the University of Aarhus, the Danish Diabetes Association and the Danish Medical Research Council.

Duality of interest S. P. Johnsen and J. Rungby have previously received an unrestricted research grant from Servier. J. Rungby and H. T. Horsdal are former consultants for Sanofi-Aventis. J. Rungby has served on advisory boards for Sanofi-Aventis and Novo Nordisk. F. Søndergaard has no duality of interest to report.

\section{References}

1. Turner RC, Cull CA, Frighi V, Holman RR (1999) Glycemic control with diet, sulfonylurea, metformin, or insulin in patients with type 2 diabetes mellitus: progressive requirement for multiple therapies (UKPDS 49). UK Prospective Diabetes Study (UKPDS) Group. JAMA 281:2005-2012

2. Malmberg K, Yusuf S, Gerstein HC et al (2000) Impact of diabetes on long-term prognosis in patients with unstable angina 
and non-Q-wave myocardial infarction: results of the OASIS (Organization to Assess Strategies for Ischemic Syndromes) Registry. Circulation 102:1014-1019

3. Meinert CL, Knatterud GL, Prout TE, Klimt CR (1970) A study of the effects of hypoglycemic agents on vascular complications in patients with adult-onset diabetes. II. Mortality results. Diabetes 19(Suppl):789-830

4. Davis TM, Parsons RW, Broadhurst RJ, Hobbs MS, Jamrozik K (1998) Arrhythmias and mortality after myocardial infarction in diabetic patients. Relationship to diabetes treatment. Diabetes Care 21:637-640

5. Inzucchi SE, Masoudi FA, Wang Y et al (2005) Insulin-sensitizing antihyperglycemic drugs and mortality after acute myocardial infarction: insights from the National Heart Care Project. Diabetes Care 28:1680-1689

6. Meier JJ, Deifuss S, Klamann A, Schmiegel W, Nauck MA (2003) Influence of an antidiabetic treatment with sulfonylurea drugs on long-term survival after acute myocardial infarction in patients with type 2 diabetes. The LAngendreer Myocardial infarction and Blood glucose in Diabetic patients Assessment (LAMBDA). Exp Clin Endocrinol Diabetes 111:344-350

7. Johnsen SP, Monster TB, Olsen ML et al (2006) Risk and shortterm prognosis of myocardial infarction among users of antidiabetic drugs. Am J Ther 13:134-140

8. Garratt KN, Brady PA, Hassinger NL, Grill DE, Terzic A, Holmes DR Jr (1999) Sulfonylurea drugs increase early mortality in patients with diabetes mellitus after direct angioplasty for acute myocardial infarction. J Am Coll Cardiol 33:119-124

9. Gustafsson I, Hildebrandt P, Seibaek M et al (2000) Long-term prognosis of diabetic patients with myocardial infarction: relation to antidiabetic treatment regimen. The TRACE Study Group. Eur Heart J 21:1937-1943

10. Fisman EZ, Tenenbaum A, Benderly M, Goldbourt U, Behar S, Motro M (1999) Antihyperglycemic treatment in diabetics with coronary disease: increased metformin-associated mortality over a 5-year follow-up. Cardiology 91:195-202

11. Halkin A, Roth A, Jonas M, Behar S (2001) Sulfonylureas are not associated with increased mortality in diabetics treated with thrombolysis for acute myocardial infarction. J Thromb Thrombolysis $12: 177-184$

12. McGuire DK, Newby LK, Bhapkar MV et al (2004) Association of diabetes mellitus and glycemic control strategies with clinical outcomes after acute coronary syndromes. Am Heart J 147:246-252

13. Murcia AM, Hennekens CH, Lamas GA et al (2004) Impact of diabetes on mortality in patients with myocardial infarction and left ventricular dysfunction. Arch Intern Med 164:2273-2279

14. Fisman EZ, Tenenbaum A, Boyko V et al (2001) Oral antidiabetic treatment in patients with coronary disease: time-related increased mortality on combined glyburide/metformin therapy over a 7.7year follow-up. Clin Cardiol 24:151-158
15. Frank L (2000) Epidemiology. When an entire country is a cohort. Science 287:2398-2399

16. Pedersen CB, Gotzsche H, Moller JO, Mortensen PB (2006) The Danish Civil Registration System. A cohort of eight million persons. Dan Med Bull 53:441-449

17. Andersen TF, Madsen M, Jørgensen J, Mellemkjoer L, Olsen JH (1999) The Danish National Hospital Register. A valuable source of data for modern health sciences. Dan Med Bull 46:263-268

18. Tunstall-Pedoe H, Kuulasmaa K, Amouyel P, Arveiler D, Rajakangas AM, Pajak A (1994) Myocardial infarction and coronary deaths in the World Health Organization MONICA Project. Registration procedures, event rates, and case-fatality rates in 38 populations from 21 countries in four continents. Circulation 90:583-612

19. Statistics Denmark (1991) IDA — an integrated database for labour market research. Statistics Denmark, Copenhagen

20. Charlson ME, Pompei P, Ales KL, MacKenzie CR (1987) A new method of classifying prognostic comorbidity in longitudinal studies: development and validation. J Chronic Dis 40:373-383

21. Steen H, Giannitsis E, Futterer S, Merten C, Juenger C, Katus HA (2006) Cardiac troponin $\mathrm{T}$ at 96 hours after acute myocardial infarction correlates with infarct size and cardiac function. J Am Coll Cardiol 48:2192-2194

22. Madsen M, Balling H, Eriksen LS (1990) The validity of the diagnosis of acute myocardial infarction in 2 registries: the Heart Registry compared to the National Patient Registry. Ugeskr Laeger 152:308-314 (article in Danish)

23. Nielsen GL, Sørensen HT, Pedersen AB, Sabroe S (1996) Analyses of data quality in registries concerning diabetes mellitus-a comparison between a population based hospital discharge and an insulin prescription registry. J Med Syst 20:1-10

24. Lachin JM, Christophi CA, Edelstein SL et al (2007) Factors associated with diabetes onset during metformin versus placebo therapy in the diabetes prevention program. Diabetes 56:1153-1159

25. Kronborg J, Johnsen SH, Njolstad I, Toft I, Eriksen BO, Jenssen T (2007) Proinsulin:insulin and insulin:glucose ratios as predictors of carotid plaque growth: a population-based 7 year follow-up of the Tromso Study. Diabetologia 50:1607-1614

26. UK Prospective Diabetes Study (UKPDS) Group (1998) Effect of intensive blood-glucose control with metformin on complications in overweight patients with type 2 diabetes (UKPDS 34). Lancet 352:854-865

27. Kao J, Tobis J, McClelland RL et al (2004) Relation of metformin treatment to clinical events in diabetic patients undergoing percutaneous intervention. Am J Cardiol 93:1347-1350

28. McAfee AT, Koro C, Landon J, Ziyadeh N, Walker AM (2007) Coronary heart disease outcomes in patients receiving antidiabetic agents. Pharmacoepidemiol Drug Saf 16:711-725

29. Sauer WH, Cappola AR, Berlin JA, Kimmel SE (2006) Insulin sensitizing pharmacotherapy for prevention of myocardial infarction in patients with diabetes mellitus. Am J Cardiol 97:651-654 